\title{
Hemodynamic Effects of Glutathione-Liganded Binuclear Dinitrosyl Iron Complex: Evidence for Nitroxyl Generation and Modulation by Plasma Albumin ${ }^{\text {s }}$
}

\author{
Taiming Liu, Meijuan Zhang, Michael H. Terry, Hobe Schroeder, Sean M. Wilson, \\ Gordon G. Power, Qian Li, Trent E. Tipple, Dan Borchardt, and Arlin B. Blood
}

Division of Neonatology, Department of Pediatrics (T.L., M.Z., A.B.B.), Department of Respiratory Care (M.H.T.), and Center for Perinatal Biology (H.S., S.M.W., G.G.P., A.B.B.), Loma Linda University School of Medicine, Loma Linda, California; Neonatal Redox Biology Laboratory, Division of Neonatology, University of Alabama at Birmingham, Birmingham, Alabama (Q.L., T.E.T.); and Department of Chemistry, University of California, Riverside, California (D.B.)

Received October 30, 2017; accepted February 21, 2018

\section{ABSTRACT}

Glutathione-liganded binuclear dinitrosyl iron complex (glutBDNIC) has been proposed to be a donor of nitric oxide (NO). This study was undertaken to investigate the mechanisms of vasoactivity, systemic hemodynamic effects, and pharmacokinetics of glut-BDNIC. To test the hypothesis that glut-BDNICs vasodilate by releasing $\mathrm{NO}$ in its reduced [nitroxyl (HNO)] state, a bioassay method of isolated, preconstricted ovine mesenteric arterial rings was used in the presence of selective scavengers of $\mathrm{HNO}$ or $\mathrm{NO}$ free radical $\left(\mathrm{NO}^{\circ}\right)$; the vasodilatory effects of glutBDNIC were found to have characteristics similar to those of an HNO donor and markedly different than an $\mathrm{NO}^{\bullet}$ donor. In addition, products of the reaction of glut-BDNIC with CPTIO [2-(4-carboxyphenyl)-4,4,5-tetramethyl imidazoline-1-oxyl-3-oxide] were found to have electron paramagnetic characteristics similar to those of an $\mathrm{HNO}$ donor compared with an $\mathrm{NO}^{\circ}$ donor. In contrast to $S$-nitroso-glutathione, which was vasodilative both in vitro and in vivo, the potency of glut-BDNIC-mediated vasodilation was markedly diminished in both rats and sheep. Wire myography showed that plasma albumin contributed to this loss of hypotensive effects, an effect abolished by modification of the cysteine-thiol residue of albumin. High doses of glut-BDNIC caused long-lasting hypotension in rats that can be at least partially attributed to its long circulating half-life of $\sim 44$ minutes. This study suggests that glutBDNIC is an HNO donor, and that its vasoactive effects are modulated by binding to the cysteine residue of plasma proteins, such as albumin.

\section{Introduction}

Nitric oxide (NO) plays an important vasodilatory role in mammals, including humans. NO synthase enzymes are the primary source of NO in the body, but its bioactivity is more complex than simple diffusion of NO from NO synthase to its site of action. Instead, evidence suggests roles for a number of NO congeners that are capable of both preserving and regulating its bioactivity. Many derivatives of NO, such as dinitrosyl iron complexes (DNICs) (Mulsch et al., 1991; Suryo Rahmanto et al., 2012; Vanin, 2016), S-nitrosothiols (SNOs) (Pawloski et al., 2001), and nitrite (Cosby et al., 2003), have been proposed to stabilize, store, and transport NO bioactivity.

These experiments were supported by National Heart, Lung, and Blood Institute [Grants R01HL95973 and R01HL119280], Eunice Kennedy Shriver National Institute of Child Health and Human Development [Grants P01 HD-31226 and R03HD069746], and National Science Foundation Division of Biological Infrastructure [Grant MRI-DBI 0923559]

https://doi.org/10.1124/mol.117.110957.

S This article has supplemental material available at molpharm. aspetjournals.org.
Research on DNICs is limited in comparison with extensive studies on SNOs and nitrite, despite recent evidence that DNICs are the most prevalent intracellular NO adduct (Hickok et al., 2011).

Dinitrosyl iron complexes consist of an $\mathrm{Fe}(\mathrm{NO})_{2}$ nucleus attached to anionic ligands. These ligands are often thiols and can range in size from small molecules, such as L-cysteine and glutathione, to high molecular weight proteins with thiol groups, such as albumin (Vanin, 2009). DNICs can exist with either one or two $\mathrm{Fe}(\mathrm{NO})_{2}$ nuclei, termed mononuclear (MDNIC) and binuclear (BDNIC) DNICs, respectively (Supplemental Fig. 1A) (Borodulin et al., 2014).

When applied to isolated arteries, low molecular weight MDNICs and BDNICs synthesized with glutathione or L-cysteine ligands demonstrate vasodilatory potencies comparable to that of NO itself (Vanin et al., 1996, 2007; Blum-Johnston et al., 2016). Although DNIC is capable of activating isolated soluble guanylate cyclase (sGC) (Severina et al., 2003), the mechanism by which these compounds deliver NO moiety across the plasma membrane is not known. Even the redox state of the

ABBREVIATIONS: AS, Angeli's salt; BDNIC, binuclear dinitrosyl iron complex; CPTIO, 2-(4-carboxyphenyl)-4,4,5-tetramethyl imidazoline-1-oxyl-3oxide; DNIC, dinitrosyl iron complex; DTT, 1,4-dithiothreitol; EPR, electron paramagnetic resonance; glut-BDNIC, glutathione-liganded binuclear dinitrosyl iron complex; GSH, glutathione; GSNO, S-nitroso-glutathione; HMW, high molecular weight; HNO, nitroxyl; LMW, low molecular weight; MDNIC, mononuclear dinitrosyl iron complex; $\mathrm{NO}$, nitric oxide; $\mathrm{NO}^{+}$, nitrosonium; $\mathrm{NO}^{*}$, free radical nitric oxide; $\mathrm{ODQ}, 1 \mathrm{H}-[1,2,4] \mathrm{oxadiazolo}$ [4,3-a]quinoxaline-1-one; RBC, red blood cell; sGC, soluble guanylate cyclase; SNO, S-nitrosothiol; SOD, superoxide dismutase 1. 
NO moiety in DNICs is uncertain. There are three different redox states of $\mathrm{NO}$ : nitrosonium $\left(\mathrm{NO}^{+}\right)$, free radical $\mathrm{NO}\left(\mathrm{NO}^{\circ}\right)$, and nitroxyl (HNO), each with similar but also distinctive biochemical properties (Stamler et al., 1992; Flores-Santana et al., 2011; Shoman and Aly, 2016). X-ray crystallography studies have indicated the NO moieties of DNICs are partially negatively charged (Tsai et al., 2015), suggesting they may release HNO. However, others have proposed that DNICs exist with a partial positive charge on the NO moiety, in which case the DNICs would be more likely to release $\mathrm{NO}^{\bullet}$ or even $\mathrm{NO}^{+}$that would be able to $S$-nitrosate cysteine thiols to make SNOs (Vanin, 2009; Borodulin et al., 2014), which are also potent vasodilators. Determination of the redox state of the NO species released by DNICs is a necessary step toward understanding their mechanism of bioactivity.

In addition to interests in the physiologic role of DNICs, their NO-mimetic properties have made them important therapeutic candidates (Vanin, 2009). Unlike other nitrodilators, glutathione-liganded BDNIC (glut-BDNIC) has long-lasting hypotensive effects and is currently in clinical trials for treatment of hypertension (Chazov et al., 2012). It has been proposed that glut-BDNIC is converted into more stable plasma albumin-liganded MDNIC in the circulation (Boese et al., 1995; Timoshin et al., 2007; Suryo Rahmanto et al., 2012). Nevertheless, the kinetics and mechanism of this conversion are not clear, let alone the effects on BDNICmediated vasodilation.

This study was undertaken to investigate the mechanism of glut-BDNIC-mediated vasodilation in both isolated arteries and in vivo, and to characterize the relationship between the hemodynamic effects and pharmacokinetics of glut-BDNIC. Using both wire myography bioassay and chemical analysis, we tested the hypothesis that DNICs release HNO rather than $\mathrm{NO}^{*}$. Using wire myography again, we characterized the role of plasma albumin in modulating the vasodilatory effects of glut-BDNIC. Finally, we investigated the hemodynamic effects of glut-BDNIC and correlated this with its pharmacokinetics both in vitro and in vivo.

\section{Materials and Methods}

Experimental Animals. All animal protocols were preapproved by the Institutional Animal Care and Use Committee of Loma Linda University, and were in accordance with guidelines of the American Physiologic Society and the National Institutes of Health.

Preparation of $S$-Nitroso-Glutathione, DNICs, and NO Gas. S-nitroso-glutathione (GSNO) and DNICs were prepared as reported previously (Borodulin et al., 2013b; Liu et al., 2016a). NO gas was generated by reaction of $\mathrm{HCl}$ and nitrite followed by purification with $\mathrm{NaOH}$. Further details are provided in the Supplemental Materials.

Surgical Procedures and Bolus Injection Protocol in Sheep. Neutered male sheep 9-10 months old and weighing $37 \pm 2 \mathrm{~kg}$ were surgically instrumented as previously reported (Liu et al., 2016a) and are described in more detail in the Supplemental Materials. After surgical instrumentation, isoflurane $(1.5 \%-2.5 \%)$ was discontinued, and anesthesia was maintained with ketamine $(1 \mathrm{mg} / \mathrm{kg})$ and vecuronium $(0.1 \mathrm{mg} / \mathrm{kg}$; Sun Pharmaceutical, Mumbai, India) given intravenously and supplemented hourly or as needed. L-N $\mathrm{N}^{\mathrm{G}}$-nitro arginine methyl ester $(45 \mathrm{mg} / \mathrm{kg})$ and hexamethonium $(1 \mathrm{mg} / \mathrm{kg}$ for initial dose; $2 \mathrm{mg} \cdot \mathrm{h}^{-1} \cdot \mathrm{kg}^{-1}$ for continuous dose) were given intravenously to block endogenous NO synthesis and sympathetic activity, respectively. After a baseline period of 30 minutes, a 1-ml bolus of NO provided by dissolving NO gas in saline, GSNO, or glut-BDNIC was injected into the jugular vein while the mean arterial blood pressure was recorded through a catheter in the brachial artery. Average hypotensive responses during 120 -second intervals were calculated and normalized to the baseline values.

Surgical Procedures and Experimental Protocol in Rats. Female rats weighing $301 \pm 3 \mathrm{~g}$ were anesthetized and surgically prepared in a manner similar to the sheep and as described in the Supplemental Materials. After surgical instrumentation, isoflurane (2.5\%) was discontinued, and anesthesia was maintained with an intraperitoneal injection of urethane $(1000 \mathrm{mg} / \mathrm{kg})$ and supplemented thereafter as required. Hexamethonium $(1 \mathrm{mg} / \mathrm{kg}$ for initial dose; $2 \mathrm{mg} \cdot \mathrm{h}^{-1} \cdot \mathrm{kg}^{-1}$ for continuous dose) was given intravenously to limit neural influences. Mean arterial blood pressure and heart rate were recorded through a catheter in the carotid artery. Rats were given continuous infusions or bolus injections. In the continuous infusion protocol, $25 \mu \mathrm{M}$ glut-BDNIC or $50 \mu \mathrm{M}$ GSNO was infused into the lower abdominal aorta at rates of $0.05,0.1,0.2$, and $0.4 \mathrm{ml} / \mathrm{min}$, increasing every 3 minutes, with doses providing a total of $\sim 0.19$ $\mu \mathrm{mol} / \mathrm{kg}$ glut-BDNIC or $\sim 0.37 \mu \mathrm{mol} / \mathrm{kg}$ GSNO, respectively. Since each glut-BDNIC molecule contains four NO moieties, this equates to $\sim 0.75 \mu \mathrm{mol} / \mathrm{kg}$ NO moiety during the 12 -minute infusion. In the bolus injection protocol, $0.29 \pm 0.02 \mathrm{ml}$ of $2.5 \mathrm{mM}$ glut-BDNIC $(2.42 \mu \mathrm{mol} / \mathrm{kg}$ glut-BDNIC molecule or $9.68 \mu \mathrm{mol} / \mathrm{kg}$ NO moiety) was injected into the lower abdominal aorta over 5 seconds. Blood samples were then collected from the jugular vein for electron paramagnetic resonance (EPR) analyses. Average hemodynamic responses during 20-second intervals at different time points were calculated and compared with baseline values.

Wire Myography. Ovine mesenteric arterial rings (2-mm diameter and $5 \mathrm{~mm}$ long) were denuded of endothelium (verified with $1 \mu \mathrm{M}$ acetylcholine) and mounted in organ bath chambers as previously described (Liu et al., 2016b). Following preconstriction with $10 \mu \mathrm{M}$ serotonin, dose-response curves were measured for study drugs including glut-BDNIC, glut-MDNIC, PROLI-NONOate (NO` donor; Cayman Chemical, Ann Arbor, MI), GSNO, and Angeli's salt (AS; HNO donor; Cayman Chemical). Test compounds were added to the baths 5 minutes before the contraction and maintained until the end of the experiments. These compounds included the SGC inhibitor ODQ (1H-[1,2,4] oxadiazolo[4,3-a]quinoxaline-1-one; $10 \mu \mathrm{M})$, the $\mathrm{NO}^{\bullet}$ and HNO scavenger CPTIO [2-(4-carboxyphenyl)-4,4,5-tetramethyl imidazoline-1-oxyl-3-oxide; $200 \mu \mathrm{M}$ ], superoxide dismutase 1 (SOD; $1000 \mathrm{U} / \mathrm{ml}), 1$,4-dithiothreitol (DTT; $1 \mathrm{mM})$, bovine albumin $(15 \mu \mathrm{M})$ (all from Sigma-Aldrich, St. Louis, MO), and heparinized plasma collected from sheep. Relaxation was normalized to the tension (100\%) measured just prior to the first addition of the dose-response drug. Spontaneous changes in the tension of control vessels that did not receive a test drug were subtracted from individual experiments before calculation of responses. Maximal absolute tensions of sheep mesenteric arterial rings in the absence and presence of different test compounds are shown in Supplemental Fig. 2.

Hydroxylamine Assay. The details are described in the Supplemental Materials.

EPR. EPR signals were recorded from paramagnetic DNICs at room temperature or $150 \mathrm{~K}$ using an X-Band EPR spectrometer (Bruker, Billerica, MA). Samples with CPTIO $(50 \mu \mathrm{M})$ were analyzed on site at room temperature, whereas other samples were snap-frozen in quartz tubes (707-SQ-250M, Wilmad LabGlass; SP Industries, Warminster, PA) in liquid nitrogen and kept at $-80^{\circ} \mathrm{C}$ until analyses. The EPR was set to a microwave power of $20 \mathrm{~mW}$ and modulation amplitude of $0.1 \mathrm{G}$ for room temperature measurements, and $2 \mathrm{~mW}$ and $1.0 \mathrm{G}$, respectively, for measurements at $150 \mathrm{~K}$. A standard curve of glut-MDNIC (1-50 $\mu \mathrm{M})$ was made daily. Whereas glut-BDNIC per se was EPR-silent (EPR unmeasurable), the metabolite formed in the presence of plasma or albumin had an EPR spectra similar to that of MDNICs and was quantified using the standard curve of glut-MDNIC. The intensity of the EPR signal, an index of the amount of unpaired electrons, was quantified by double integration using WIN-EPR (Bruker). 

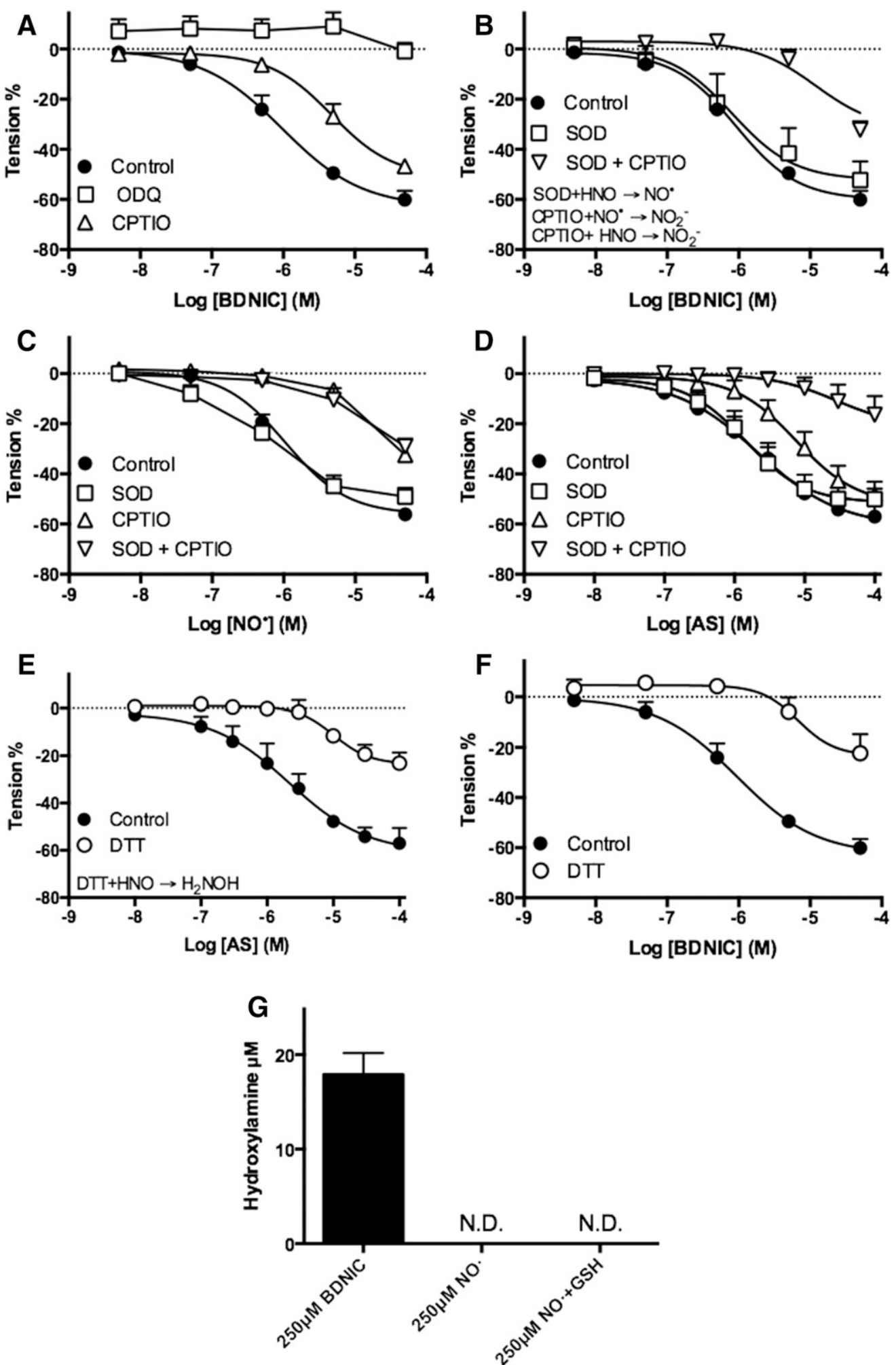

Fig. 1. Role of HNO in BDNIC-mediated relaxation in isolated sheep mesenteric arteries $[n \geq 5$ for (A-F); $n=3$ for $(\mathrm{G})]$. Glut-BDNIC-mediated relaxation in the absence and presence of the sGC inhibitor ODQ $(10 \mu \mathrm{M})$ or the NO ${ }^{\bullet}$ and HNO scavenger CPTIO (200 $\left.\mu \mathrm{M}\right)(\mathrm{A})$, or the enzyme SOD $\left(1000 \mathrm{U} / \mathrm{ml}\right.$, converts HNO into NO ${ }^{\circ}$ or SOD $(1000 \mathrm{U} / \mathrm{ml})$ and CPTIO $(200 \mu \mathrm{M})(\mathrm{B})$. Effects of SOD $(1000 \mathrm{U} / \mathrm{ml})$, CPTIO $(200 \mu \mathrm{M})$, or SOD $(1000 \mathrm{U} / \mathrm{ml})$ and CPTIO $(200 \mu \mathrm{M})$ on $\mathrm{NO}^{\circ}$ - (from PROLI-NONOate) $(\mathrm{C})$ and HNO-mediated (from AS) relaxation (D). The effects of DTT (1 mM, HNO scavenger) on AS- (E) and glut-BDNIC-mediated (F) relaxation. The vessel rings were $\sim 2 \mathrm{~mm}$ in diameter and $5 \mathrm{~mm}$ long. Information concerning the various reactions is shown in each panel. Relaxation was normalized to the tension $(100 \%)$ prior to the first addition of the dose-response drug. (G) In vitro measurements to detect the production of hydroxylamine, a metabolite of HNO, from glut-BDNIC. N.D., not detected. 
Modification of Albumin. To test for interactions of glut-BDNIC with albumin, the protein was modified chemically. Albumin $(840 \mu \mathrm{M})$ in phosphate-buffered saline at $\mathrm{pH}=7.4$ was incubated with the thiol modifier $\mathrm{HgCl}_{2}(5 \mathrm{mM})$ and/or the histidine modifier diethylpyrocarbonate $(5 \mathrm{mM})$ at room temperature for 40 minutes and then desalted using a Sephadex G-25 column with an exclusion limit of $5000 \mathrm{M}_{\mathrm{r}}$ (GE Healthcare, Uppsala, Sweden).

Statistics. Average values are given as the mean \pm S.E.M. in the text and figures. Statistical analyses were carried out with Prism, version 5.0c (GraphPad Software, La Jolla, CA), with significance accepted at $P<0.05$. One-way analysis of variance with Dunnett's test was used to test significance of changes associated with rates of intravenous infusion. Student's $t$ test was used where indicated. Relaxation results from wire myography experiments were quantified and compared by fitting the data to the Gaddum/Schild model in Prism. Rates of DNIC elimination from circulating blood were quantified by fitting the concentration versus time curve to a onephase decay function using Prism after subtraction of baseline concentrations (nil).

\section{Results}

Verification of Laboratory-Prepared DNICs. The UV-Vis spectra of glut-BDNIC and glut-MDNIC were as others have found (Borodulin et al., 2014), indicating successful synthesis (Supplemental Fig. S1). Glut-MDNIC displayed a typical EPR signal with an axially symmetric g-factor with $\mathrm{g}_{\perp}=2.04, \mathrm{~g}_{\|}=2.014$, and $\mathrm{g}_{\mathrm{av}}=2.03$. GlutBDNIC, which itself is diamagnetic, was EPR-silent, indicating no detectable contamination with glut-MDNIC. The prepared glut-BDNIC was $99.5 \% \pm 0.7 \%$ pure as estimated by the extinction coefficient of $7400 \mathrm{M}^{-1} \mathrm{~cm}^{-1}$ at $360 \mathrm{~nm}$ (Borodulin et al., 2014).

Role of HNO in BDNIC-Mediated Relaxation. The relaxation of isolated ovine arterial rings induced by glutBDNIC was eliminated by the sGC inhibitor ODQ (Fig. 1A; does not degrade DNICs, as described in the Supplemental Materials), indicating that the relaxation is sGC-dependent (Moro et al., 1996). CPTIO, which scavenges both $\mathrm{NO}^{\bullet}$ and HNO (Goldstein et al., 2003; Samuni et al., 2010), attenuated the response to glut-BDNIC (Fig. 1A) as evidenced by a decrease in the $\mathrm{pEC}_{50}$ from $6.03 \pm 0.16$ to $5.33 \pm 0.13(P<$ 0.05). To assess whether the attenuating effects of CPTIO on glut-BDNIC-mediated vasodilation were due to $\mathrm{HNO}$ or $\mathrm{NO}^{\bullet}$ scavenging, we compared the effects of CPTIO on doseresponse curves to known donors of either HNO (AS) or NO (provided by PROLI-NONOate). Similar to its attenuation of glut-BDNIC-mediated vasodilation, CPTIO resulted in a partial right shift in the dose-response curve to HNO (Fig. $1 \mathrm{D}, \mathrm{pEC}_{50}$ from $5.7 \pm 0.2$ to $5.1 \pm 0.2$ ). In contrast, vasodilatory responses to $\mathrm{NO}^{-}$were almost completely blocked (Fig. $1 \mathrm{C}, \mathrm{pEC}_{50}$ from $6.0 \pm 0.1$ to an extent that the $\mathrm{EC}_{50}$ could not be calculated). Notably, the effect of CPTIO on vasodilation by glut-BDNIC was similar in magnitude to its effect on the AS dose-response curve, consistent with release of HNO by glut-BDNIC.

To further test the possibility that glut-BDNIC releases HNO, the effects of CPTIO were compared in the presence and absence of SOD, which readily converts $\mathrm{HNO}$ into $\mathrm{NO}^{\bullet}$ (Zeller et al., 2009). In the presence of SOD, the ability of CPTIO to attenuate vasodilation by glut-BDNIC was enhanced to a level comparable to its ability to attenuate vasodilation by $\mathrm{NO}^{\bullet}$ (Fig. $1, \mathrm{~A}$ and $\mathrm{B}, P<0.05$ at $\log [\mathrm{BDNIC}](\mathrm{M})=-5.30$ ), consistent with the idea that SOD was converting HNO to $\mathrm{NO}^{\circ}$, which is more efficiently scavenged by CPTIO. SOD potentiated the right shift caused by CPTIO for both glutBDNIC and AS, but had no effect on $\mathrm{NO}^{\circ}$-induced relaxation (Fig. 1, B-D). In further support of the involvement of HNO, the HNO scavenger DTT (Zeller et al., 2009), which significantly blocked relaxation by the HNO donor AS (Fig. 1E), also blocked glut-BDNIC-mediated relaxation (Fig. 1F). Taken together, these findings demonstrate a potent vasodilatory activity of glut-BDNIC in isolated arterial rings and suggest a role for the release of HNO.

Parallel experiments were performed using glut-MDNIC. As shown in Supplemental Fig. 3A, SOD alone did not alter glut-MDNIC-mediated relaxation, and CPTIO only partially inhibited it. However, the inhibitory effects of CPTIO were potentiated by the presence of SOD. Furthermore, glutMDNIC-mediated vasodilation was blocked by ODQ and significantly attenuated by DTT (Supplemental Fig. 3B). These parallel findings are all consistent with glut-MDNIC acting as an HNO donor in a manner similar to glut-BDNIC.

Chemical Verification of Glut-DNICs as HNO Donor. To further test the hypothesis that glut-DNIC releases HNO, experiments were conducted based on the recently reported distinction between the reactions of $\mathrm{HNO}$ and $\mathrm{NO}^{\circ}$ with CPTIO (Samuni et al., 2010), which demonstrated that EPR can be used to distinguish between the products of CPTIO reacting with either $\mathrm{NO}^{\bullet}$ or $\mathrm{HNO}$. Consistent with previous reports (Samuni et al., 2010), we found CPTIO to have a fivepeak EPR spectrum (Fig. 2A). This spectrum was rapidly converted to a seven-peak spectrum upon reaction of the CPTIO with NO* provided by the NO donor PROLI-NONOate (Fig. 2B). In contrast, the five-peak CPTIO spectrum gradually disappeared over a period of 90 minutes if the CPTIO was allowed to react with HNO from excess AS (Fig. 2D). Finally, addition of an oxidizing solution of $\mathrm{CuSO}_{4} / \mathrm{H}_{2} \mathrm{O}_{2} /$ ferricyanide $(10 \mu \mathrm{M} / 10 \mathrm{mM} / 1 \mathrm{mM})$ resulted in the seven-peak spectrum (Fig. 2D). These findings are consistent with previous work characterizing the EPR spectra of CPTIO products of reaction with $\mathrm{NO}^{\bullet}$ or $\mathrm{HNO}$, as well as oxidation of the product of the reaction of CPTIO with HNO (Samuni et al., 2010). Importantly, similar to AS, addition of glut-BDNIC to CPTIO resulted in a complete loss of the EPR spectrum, which could be restored to the seven-peak spectra by the addition of $\mathrm{CuSO}_{4} / \mathrm{H}_{2} \mathrm{O}_{2}$ /ferricyanide (Fig. 2E). Notably, glutathione (GSH) alone at a comparable level did not eliminate the EPR signal of CPTIO (Fig. 2C), indicating that the effect of glutBDNIC on the CPTIO spectra was not due to GSH contamination. Taken together, these results are consistent with the idea that glut-BDNIC releases $\mathrm{HNO}$, not $\mathrm{NO}^{\circ}$. In addition, the EPR signal of CPTIO was also eliminated by glut-MDNIC (data not shown), suggesting the same conclusion may be applied to glut-MDNIC.

Further experiments were performed to characterize the NO species released from glut-BDNIC. Using HEPES buffer $(\mathrm{pH}$ 7.4) as a reagent in a purge vessel in line with a chemiluminescence $\mathrm{NO}^{\bullet}$ analyzer, the $\mathrm{NO}^{\bullet}$ release from glutBDNIC was compared with that from the $\mathrm{NO}^{\bullet}$ and $\mathrm{HNO}$ donors. Similar to AS but different from PROLI-NONOate, glut-BDNIC released minimal amounts of $\mathrm{NO}^{*}$. These results were not affected by the presence of isolated mesenteric artery tissue in the purge vessel (Supplemental Fig. 4), indicating glut-BDNIC is a poor donor of $\mathrm{NO}^{\circ}$. To further investigate the 


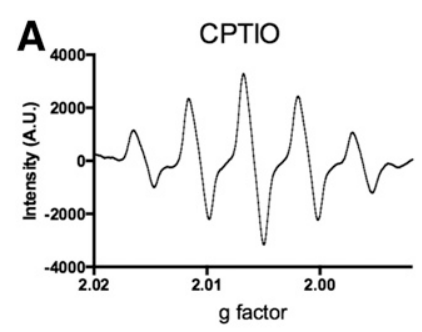

D CPTIO+AS

B

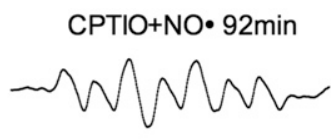

C

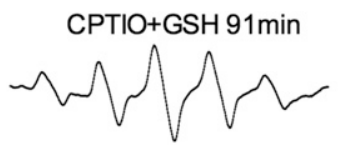

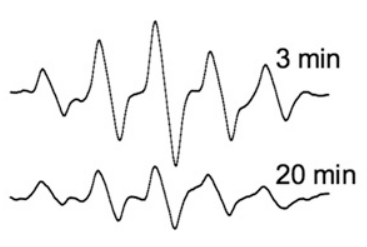
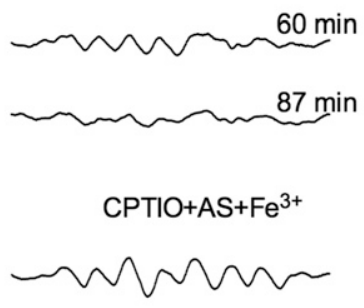

E CPTIO+BDNIC
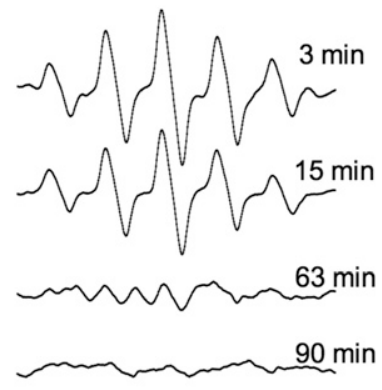

CPTIO+BDNIC+Fe ${ }^{3+}$

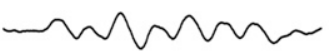

Fig. 2. Confirmation of glut-BDNIC as an HNO donor by EPR, utilizing the difference between $\mathrm{HNO}$ and $\mathrm{NO}^{\circ}$ reactions with CPTIO. EPR spectra of $50 \mu \mathrm{M}$ CPTIO alone (A), $50 \mu \mathrm{M}$ CPTIO $+200 \mu \mathrm{M}$ $\mathrm{NO}^{\bullet}$ (B), $50 \mu \mathrm{M}$ CPTIO + $100 \mu \mathrm{M}$ GSH (C), $50 \mu \mathrm{M}$ CPTIO $+200 \mu \mathrm{M}$ AS (D), and $50 \mu \mathrm{M}$ CPTIO $+50 \mu \mathrm{M}$ glut-BDNIC (E) at different time points as indicated above in each spectrum. The spectrum for (A) was stable for at least 3 hours. The bottom spectra in (D) and (E) were obtained after oxidation with $\mathrm{CuSO}_{4} / \mathrm{H}_{2} \mathrm{O}_{2} /$ ferricyanide (indicated as " $+\mathrm{Fe}^{3+»)}$. A representative spectrum from at least three individual experiments is shown. All experiments were performed in HEPES buffer $(\mathrm{pH}=$ 7.4). All spectra were recorded at room temperature and are shown in the same scale as that in (A). oxidative state of NO species released from glut-BDNIC, measurements of hydroxylamine, one product of HNO reduction, were obtained following incubation of glut-BDNIC in phosphate-buffered saline at $\mathrm{pH}$ 6.8. After 5-minute incubation at $95^{\circ} \mathrm{C}, 20 \mu \mathrm{M}$ hydroxylamine was formed from an initial concentration of $250 \mu \mathrm{M}$ glut-BDNIC. In contrast, levels of hydroxylamine were undetectable following incubation of $\mathrm{NO}^{\bullet}$ or GSNO (for the test of $\mathrm{NO}^{\bullet}+\mathrm{GSH}$ ) (Fig. 1G). These results are again consistent with the possibility that glut-BDNIC is a donor of HNO rather than $\mathrm{NO}^{\circ}$.

Glut-BDNIC Loses Hypotensive Effects In Vivo. Contrary to the potent relaxation found in isolated arteries in the vessel baths (Fig. 1), glut-BDNIC did not reduce blood pressure when low doses were given by continuous infusion in rats (Fig. 3A). On the other hand, GSNO given at comparable doses was vasodilative both in vivo and in vitro (Figs. $3 \mathrm{~B}$ and $4 \mathrm{E}$ ). Similar results were obtained in sheep given low doses of glut-BDNIC or GSNO by bolus injection (Fig. 4A). The hypotensive responses to bolus injections of $\mathrm{NO}^{\bullet}$, GSNO, and glut-BDNIC in sheep are shown in Fig. 4, B-D. One possible reason for the lack of response in vivo is that that glutBDNIC is converted in circulating blood into a form that does not release $\mathrm{HNO}$ as readily. Another possibility is that any $\mathrm{HNO}$ that might be released from glut-BDNIC is rapidly scavenged by reaction with other components present in the blood but not in the vessel baths. Notably, the observation that $50 \mu \mathrm{M}$ GSNO caused vasodilation at $0.1 \mathrm{ml} / \mathrm{min}$ whereas $25 \mu \mathrm{M}$ glut-BDNIC (which contains $100 \mu \mathrm{M}$ NO moieties) infused at $0.4 \mathrm{ml} / \mathrm{min}$ had no effect (Fig. 3) suggests that the production of SNOs from
glut-BDNIC in vivo, if it occurs at all, accounts for less than 1/8 of the NO moieties in glut-BDNIC.

Role of Plasma Albumin in the Loss of Hypotensive Effects of Glut-BDNIC. Further tests were carried out in isolated arteries to learn what component of blood might prevent glut-BDNIC-induced vasodilation. The iron chelators pyridoxal isonicotinoyl hydrazone and EDTA (ethylene diamine tetraacetic acid) did not affect the glut-BDNICmediated relaxation (Fig. 5B), indicating no role for endogenous iron chelators. Addition of heparinized plasma to the vessel bath significantly right shifted the dose-response curve of glut-BDNIC $(P<0.05$; Fig. 5A), indicating that one of its components contributes to the loss of hypotensive effects of glut-BDNIC in vivo (Fig. 3A). Similar to plasma, albumin alone right shifted the dose-response curve of glut-BDNIC significantly $(P<0.05$; Fig. $5 \mathrm{C})$, indicating it plays a role, perhaps by either scavenging $\mathrm{HNO}$ or stabilizing the BDNIC and thus inhibiting its release of HNO. The first possibility was tested and ruled out by the finding that plasma did not significantly attenuate AS-mediated relaxation (Supplemental Fig. 5). To test whether the histidine or cysteine residues of albumin were involved in stabilization of glut-BDNIC, experiments were carried out with covalently modified albumin. Modification of histidine residues resulted in no change in the attenuating effects of albumin on BDNIC-mediated relaxation (Fig. 5D). Modification of the cysteine-thiols, however, resulted in a loss of the attenuating effects of albumin $(P<0.05$; Fig. 5D). These results indicate that the cysteine-thiol, but not the histidine
A

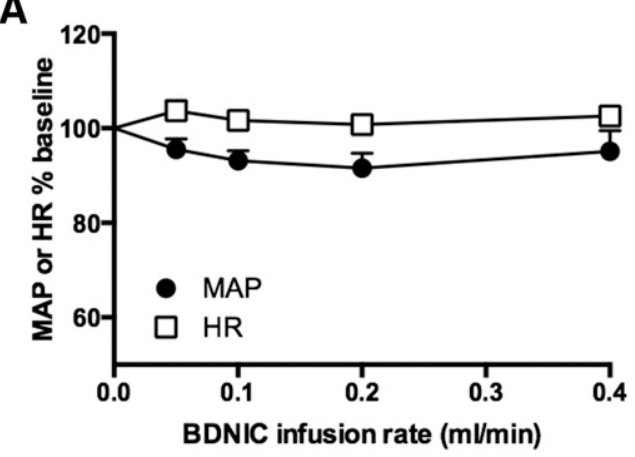

B

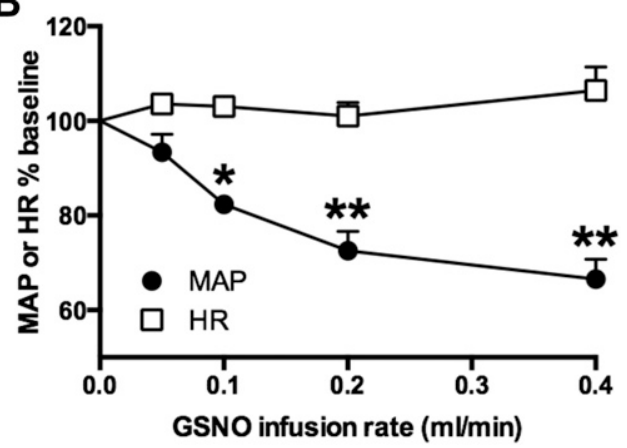

Fig. 3. Effects of glut-BDNIC (A) and GSNO (B) on mean arterial blood pressure (MAP) and heart rate (HR) in anesthetized rats during continuous, stepped intravascular infusions $(n \geq 5)$. Twenty-five micromolar glutBDNIC (equivalent to $100 \mu \mathrm{M}$ NO moieties) or $50 \mu \mathrm{M}$ GSNO (equivalent to $50 \mu \mathrm{M}$ NO moieties) was infused at increasing rates with each rate maintained for 3 minutes. $* P<0.05$; ** $P<$ 0.01 vs. baseline (100\%); one-way analysis of variance with Dunnett's post hoc test. 

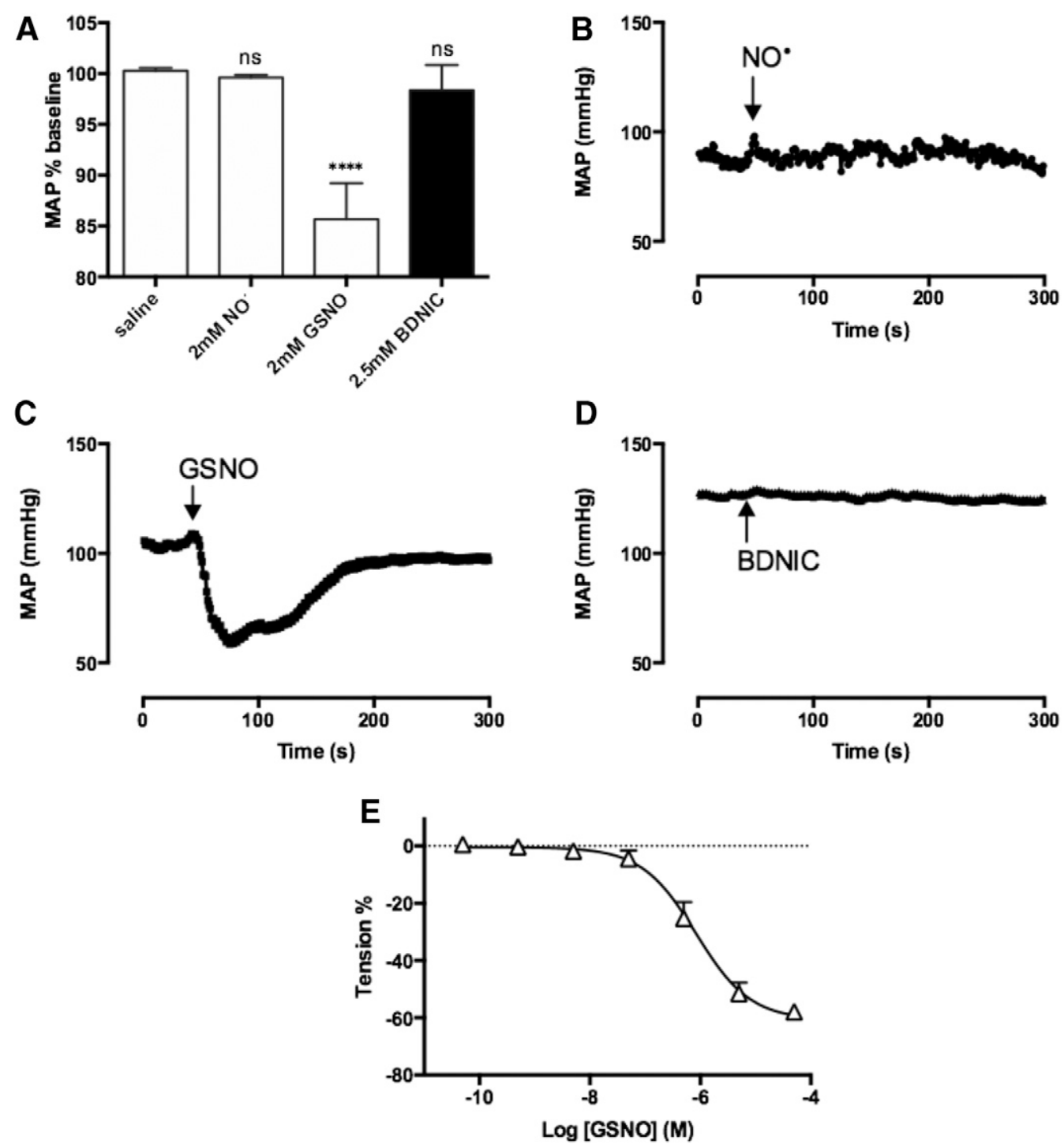

Fig. 4. Hypotension induced by bolus injections of $\mathrm{NO}^{*}$, GSNO, and glut-BDNIC in anesthetized sheep. (A) Effects of NO, GSNO, and glut-BDNIC on mean arterial blood pressure (MAP) following a 1.0-ml bolus given in the jugular vein $(n \geq 4)$. (B-D) Representative hypotensive effects of bolus injection of NO (1 ml of $2.0 \mathrm{mM}$ or $54 \mathrm{nmol} / \mathrm{kg}$ ) (B), GSNO (1 ml of $2 \mathrm{mM}$ or $54 \mathrm{nmol} / \mathrm{kg})(\mathrm{C})$, and glut-BDNIC (1 ml of $2.5 \mathrm{mM} \mathrm{or} 270 \mathrm{nmol} / \mathrm{kg}$ ) (D). (E) Relaxation in isolated sheep mesenteric arteries in response to GSNO $(n=5)$. **** $P<0.0001$ vs. saline (one-way analysis of variance with Dunnett's post hoc test). ns, not significant.

residue, in albumin plays an important role in attenuating the vasodilatory effects of glut-BDNICs. Whole blood contracted the isolated arterial rings significantly, and thus its effects on glut-BDNIC-mediated relaxation were not tested.

Parallel experiments were performed to evaluate the effects of plasma and albumin on vasodilation by glut-MDNIC. In contrast to the significant attenuating effects of plasma and albumin on glut-BDNIC-mediated vasodilation, neither plasma nor albumin significantly altered glut-MDNIC-induced relaxation (Supplemental Fig. 6), suggesting the functional effects of glut-BDNIC differ from those of glut-MDNIC under these conditions.

Long-Lasting Hypotensive Effects and Pharmacokinetics of BDNIC. Differing from low doses, high doses of glut-BDNIC led to long-lasting hypotensive effects in intact rats (Fig. 6A). Within 3 minutes after infusion of the EPR-silent glut-BDNIC, an MDNIC-like EPR signal was detected in blood samples, although a shallow shoulder appeared on the left side of the spectrum (Fig. 6C). A similar spectrum with a shallow shoulder could be observed after addition of glut-BDNIC to isolated blood, plasma, and albumin solutions (Supplemental Fig. 7). These findings suggest that glut-BDNIC is rapidly converted to an MDNIC-like species whether infused in vivo or added to blood, plasma, or albumin solutions in vitro. Glut-BDNIC retained some vasodilatory activity in vivo, albeit at markedly higher doses.

The intensity of the EPR signal at the first time point (3 minutes) following infusion of glut-BDNIC corresponded to $70.9 \pm 2.9 \mu \mathrm{M}$ MDNIC (Fig. 6D). This concentration is comparable to that which would be predicted based on a volume of distribution equal to the rat blood volume $\left[\left(4.84 \mu \mathrm{mol} \mathrm{Fe}(\mathrm{NO})_{2}\right.\right.$ nuclei infused per $\mathrm{kg} \div 0.068 \mathrm{l}$ of blood per $\mathrm{kg}=71.1 \mu \mathrm{M}$ ], assuming a blood volume in rats of $\sim 0.068$ 1/kg (Lee and Blaufox, 1985), suggesting that all glut-BDNIC administered was converted to MDNIC-like metabolites that were retained in the blood. The time course of the DNIC 

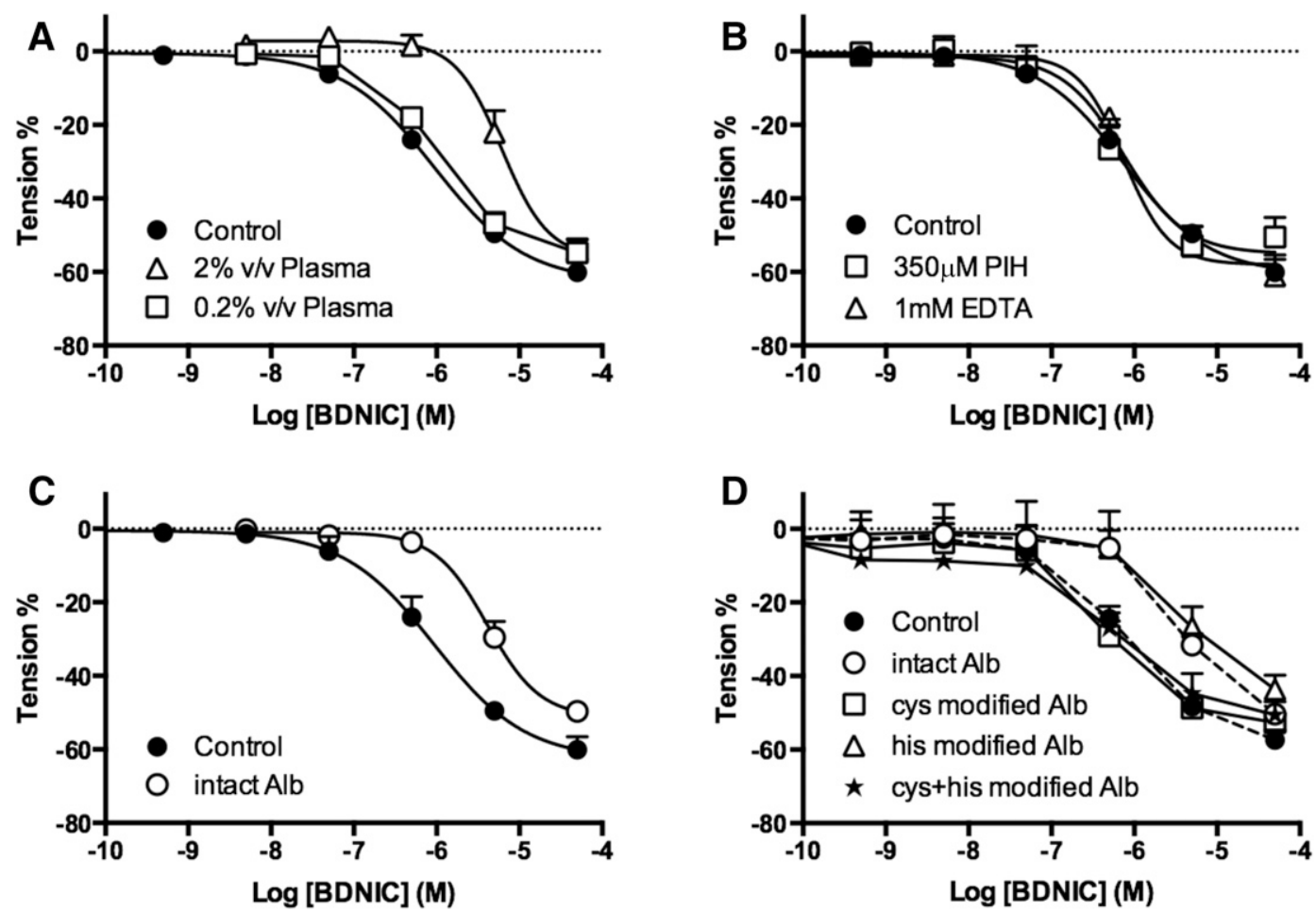

Fig. 5. Inhibition of glut-BDNIC-mediated relaxation by albumin in plasma ( $n \geq 5)$. Effects of plasma (A), chelators pyridoxal isonicotinoyl hydrazine (PIH) and EDTA (B), albumin (Alb) (C), and albumin with modified cysteine (cys) and/or histidine (his) residuals (D) on glut-BDNIC-mediated relaxation. Interrupted curves in (D) for "control" and "intact Alb" are repeats of those in (C) and were obtained in parallel with those for "modified Alb." Given that $2 \%(\mathrm{v} / \mathrm{v})$ plasma contains $\sim 12-16 \mu \mathrm{M}$ albumin, $15 \mu \mathrm{M}$ albumin was tested in $(\mathrm{C})$ and (D). Relaxation was normalized to the tension (100\%) prior to the first addition of the glut-BDNIC.

disappearance from the circulation followed first-order reaction kinetics with a half-life of $\sim 44$ minutes (Fig. 6D). Thus, the long duration of the hypotensive effects may be at least partially attributed to the relatively long elimination half-life of the MDNIC-like metabolite.

Conversion and Distribution of BDNIC in Blood In Vitro. Given the evidence that intravenously administered glut-BDNIC is rapidly and completely converted into a MDNIC-like metabolite in vivo, we sought to characterize the conversion and distribution of glut-BDNIC in blood in vitro. Addition of EPR-silent glut-BDNIC to either lysed red blood cells (RBCs) or plasma efficiently resulted in an EPRdetectable MDNIC-like metabolite. In contrast, glut-BDNIC added to the low molecular weight (LMW) fraction of plasma remained EPR-silent (Fig. 7A). Compared with native plasma, the conversion of glut-BDNIC into EPR-detectable species was about $50 \%$ less efficient in the presence of only the high molecular weight (HMW) fraction of plasma or albumin solution (Fig. 7A). Thus, the HMW fraction of plasma and the albumin therein play important roles in converting glutBDNIC to MDNIC-like metabolites, and this conversion may be facilitated by one or more components found in the LMW fraction of plasma.

Similar to plasma and lysed RBCs, whole blood converted glut-BDNIC into MDNIC-like metabolite (Fig. 7B). All of the EPR-detectable metabolite was recovered in the plasma compartment of whole blood, indicating that the conversion to MDNIC-like metabolite occurs in the plasma and not in the interior of RBCs. In comparison with whole blood, washed RBCs suspended in saline were less effective at converting glut-BDNIC (Fig. 7B) into an EPR-detectable species, in agreement with the conclusion that plasma and probably the albumin therein play important roles in the conversion. No MDNIC-like metabolite was detected in the RBCs of plasmafree blood, indicating that glut-BDNIC itself, without binding to plasma proteins, was also not membrane-permeable.

Following addition of glut-BDNIC to whole blood, most of the MDNIC-like metabolite was recovered in the HMW fraction of plasma, consistent with its binding to plasma proteins (Fig. 7C). In addition, after adding glut-BDNIC to blood, plasma, or dissolved albumin, the EPR spectra obtained at room temperature did not display an isotropic hyperfine structure (seen as multiple sharp peaks pointing upward at the left side of the spectrum versus down on the right side in the bottom spectrum of Supplemental Fig. 8), which is characteristic of LMW but not HMW MDNIC (Vanin, 2009). These results further support the conclusion that the glut-BDNIC in blood and plasma was converted to HMW complexes likely containing albumin.

\section{Discussion}

The results of the present experiments are consistent with previous work demonstrating that glut-BDNIC is a potent vasodilator via sGC activation (Vanin, 2009). However, contrary to previous propositions that DNICs are donors of $\mathrm{NO}^{\bullet}$ and $\mathrm{NO}^{+}$(Vanin, 2009; Borodulin et al., 2013a), we present both functional and chemical evidence that glut-DNIC acts by releasing HNO. In addition, our results suggest that the vasodilating potency of glut-BDNIC is markedly attenuated in the presence of plasma proteins (such as albumin), likely through an interaction with the cysteine residues. 


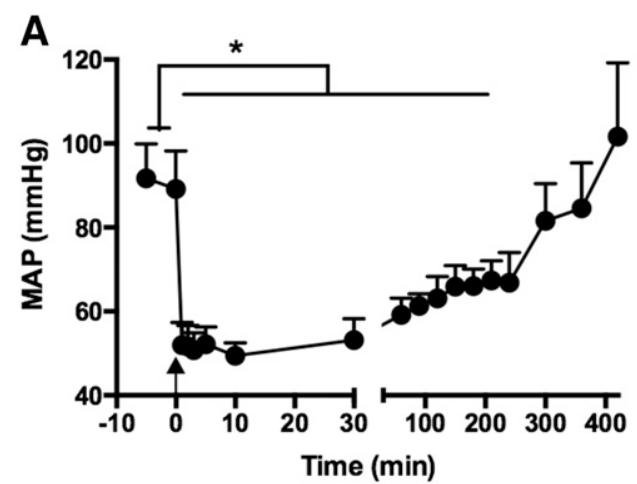

C

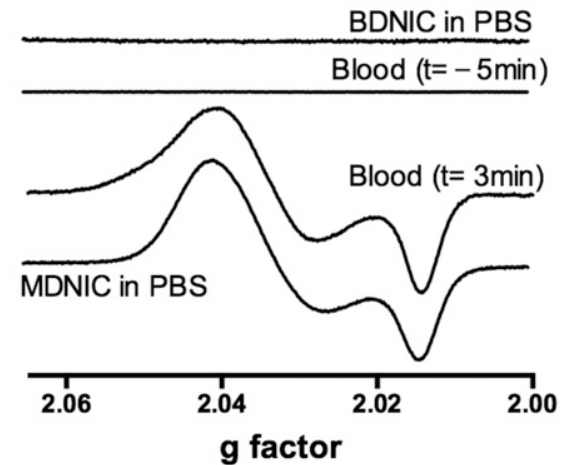

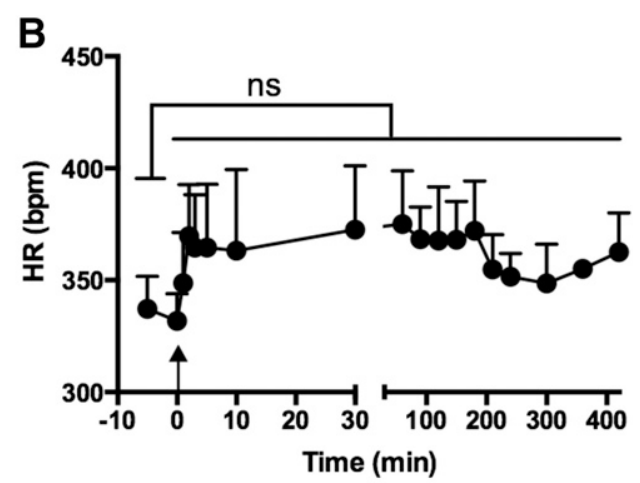

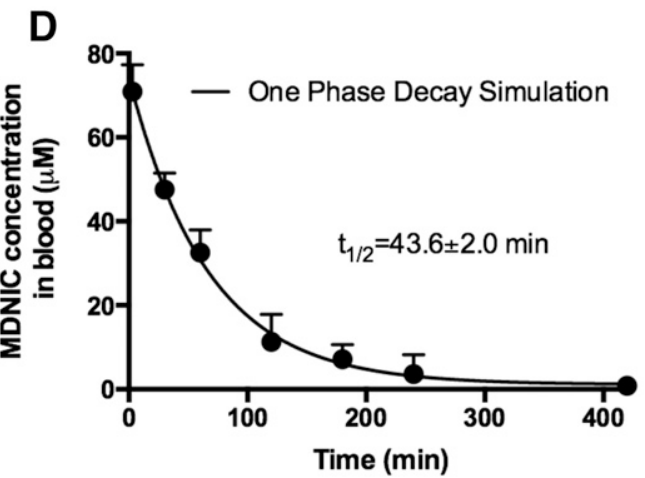

Fig. 6. Long-lasting hypotensive effects of glut-BDNIC in rats after high-dose bolus injection and pharmacokinetic findings $(n=5)$. Effects of glut-BDNIC on mean arterial blood pressure (MAP) (A) and heart rate (HR) (B) in rats after a bolus injection (shown as arrows at $0 \mathrm{minutes}$ ) at $2.42 \mu \mathrm{mol} / \mathrm{kg}(0.29 \pm$ $0.02 \mathrm{ml}$ of $2.5 \mathrm{mM} ; 9.68 \mu \mathrm{mol} / \mathrm{kg}$ NO quantity). Note the high-dose injection induces hypotensive effects lasting several hours. (C) EPR spectra (150 K) of blood samples collected 5 minutes before and 3 minutes after glut-BDNIC injection, showing a comparison with those of LMW glut-BDNIC and glutMDNIC in phosphate-buffered saline (PBS). Note the EPR-silent glut-BDNIC creates an MDNIC-like detectable EPR signal in blood after administration, suggesting it is converted to MDNIC-like metabolites. (D) Time course of disappearance of MDNIC-like metabolites in blood in vivo. The dots are measured values, whereas the line is a one-phase decay curve fit to the measured values. Values follow first-order kinetics with an apparent elimination half-life of $43.6 \pm 2.0$ minutes. ${ }^{*} P<0.05$ vs. before injection, one-way analysis of variance with Dunnett's post hoc test.

What Is the Redox State of the NO Moieties in DNIC? The redox state of NO moieties in DNICs has been uncertain. Based on X-ray studies, the $\mathrm{Fe}(\mathrm{NO})_{2}$ core has been formulated as either $\left\{\mathrm{Fe}^{\mathrm{II}}\left(\mathrm{NO}^{\bullet}\right)\left(\mathrm{NO}^{-}\right)\right\}^{9}$ or $\left\{\mathrm{Fe}^{\mathrm{III}}\left(\mathrm{NO}^{-}\right)_{2}\right\}^{9}$, in which the NO moieties have partial negative charges (Tsai et al., 2015; Tseng et al., 2015) and thus might be expected to be released in the form of HNO. Alternatively, others have proposed the core to exist as $\left\{\mathrm{Fe}^{\mathrm{I}}\left(\mathrm{NO}^{+}\right)_{2}\right\}^{7}$, representing the NO moieties as positively charged (Vanin, 2009; Borodulin et al., 2013a, 2014). The latter formulation is supported by evidence that DNICs can $S$-nitrosate thiols to produce SNOs (Boese et al., 1995; Stojanovic et al., 2004; Bosworth et al., 2009; Vanin, 2009; Vanin and Burbaev, 2011).

The current results support the idea that DNICs are donors of $\mathrm{HNO}$ rather than $\mathrm{NO}^{\circ}$ or $\mathrm{NO}^{+}$. There are multiple reasons to put forward this conclusion. First, we found that the attenuation effects of CPTIO on glut-BDNIC-mediated vasodilation are comparable to its effects on dilation by the HNO donor AS. In addition, CPTIO inhibits vasodilation by the $\mathrm{NO}^{\circ}$ donor PROLI-NONOate more efficiently than by either glutBDNIC or AS, suggesting that glut-BDNIC releases an NO species more like $\mathrm{HNO}$ than $\mathrm{NO}^{\circ}$. The addition of SOD, which converts HNO to NO (Zeller et al., 2009; Flores-Santana et al., 2011), resulted in a marked increase in the ability of CPTIO to attenuate glut-DNIC-mediated vasodilation, a phenomenon that was also observed for the HNO donor AS but not for $\mathrm{NO}^{\circ}$ itself. Likewise, vasodilation by both BDNIC and the HNO donor AS was similarly attenuated by the addition of DTT, which scavenges $\mathrm{HNO}$ by converting it to $\mathrm{H}_{2} \mathrm{NOH}$ (Zeller et al., 2009). These results are all more supportive of a role for $\mathrm{HNO}$ than NO in BDNIC-mediated relaxation. Furthermore, based on the EPR-characterized distinction between the products of HNO and NO ${ }^{\circ}$ reactions with CPTIO (Samuni et al., 2010), we chemically confirmed glut-DNIC as an HNO donor. In addition, little $\mathrm{NO}^{\bullet}$ was released from glut-BDNIC, whereas hydroxylamine, an HNO metabolite, was detected in glutBDNIC, again suggesting that glut-BDNIC is a donor of HNO rather than $\mathrm{NO}^{\circ}$. Like glut-BDNIC, similar results (Supplemental Fig. 3) from our experiments with glut-MDNIC indicate that glut-MDNIC is also a donor of HNO.

The potential sources and roles of endogenous HNO have been the subject of debate for several decades (Switzer et al., 2009). As the most abundant cellular NO adduct (Hickok et al., 2011), DNICs have been proposed to be the "working form" of endogenous NO activity (Vanin, 2016). Our evidence that DNICs can behave as HNO donors provides a potential mechanism of endogenous HNO formation and implies an important role for endogenous HNO.

In addition to evidence that DNICs release HNO, several experiments reported here speak against previous proposals that DNICs release $\mathrm{NO}^{+}$, which then might act as an $S$ nitrosating agent (Boese et al., 1995; Stojanovic et al., 2004; Bosworth et al., 2009; Vanin, 2009; Vanin and Burbaev, 2011). First, in contrast to the potent hypotensive effects of GSNO in 

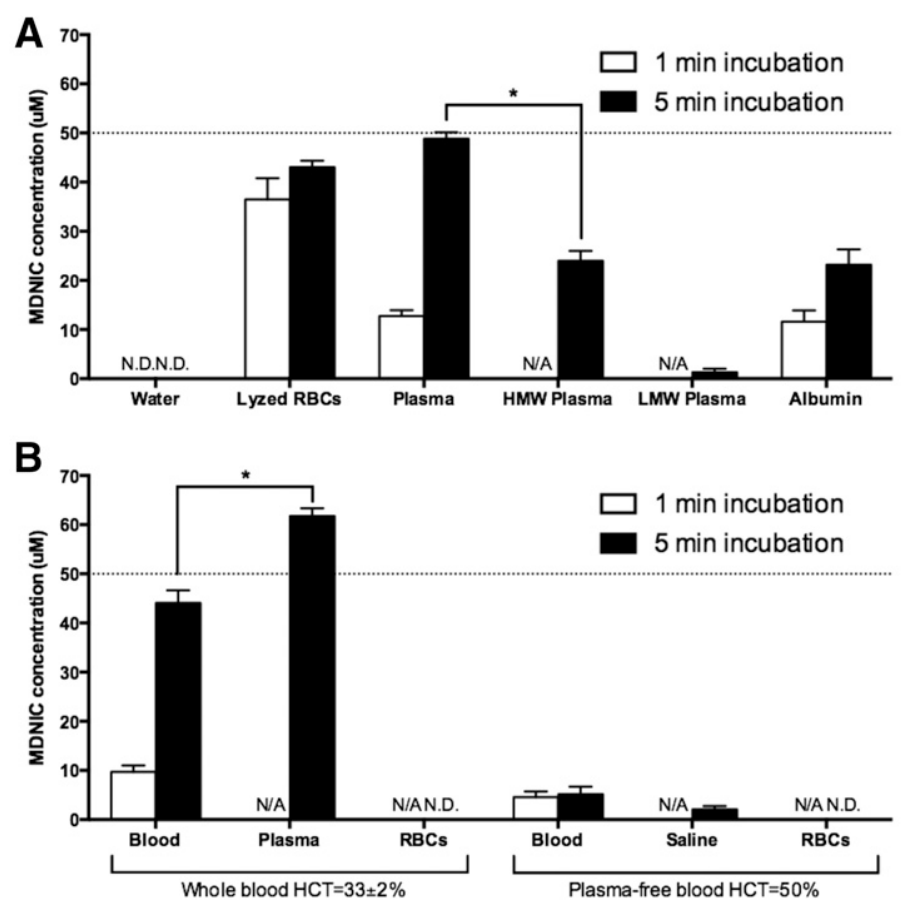
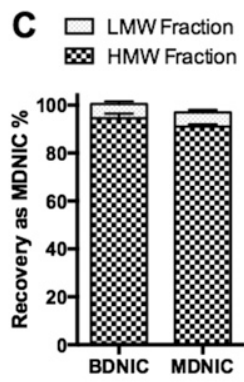

Fig. 7. Conversion and distribution of glut-BDNIC in blood in vitro $(n=5)$. (A) Conversion of EPR-silent glut-BDNIC into an EPR-detectable MDNIC-like species upon addition to water, lysed RBCs, plasma, the HMW fraction of plasma, the LMW fraction of plasma, and albumin (600 $\mu$ M) dissolved in saline. Following addition of $25 \mu \mathrm{M}$ EPR-silent glut-BDNIC into each component, the EPR-detectable MDNIC-like metabolite formed after 1 or 5 minutes of incubation $\left(37^{\circ} \mathrm{C}\right)$ was quantified using a standard curve of glut-MDNIC. Lysed RBCs: RBCs washed three times with saline, then lysed by addition of water $[1: 1(\mathrm{v} / \mathrm{v})]$ and two cycles of freeze/thaw. The HMW and LMW fractions of plasma were separated by size-exclusion chromatography (cutoff $5000 \mathrm{MW}$ ). Albumin was dissolved in saline at $600 \mu \mathrm{M}$, a concentration comparable to that of normal plasma. The horizontal dashed line at $50 \mu \mathrm{M}$ represents the level that would be expected if $100 \%$ of the $25 \mu \mathrm{M}$ glut-BDNIC were converted into MDNIC. Statistical significance was only evaluated for the 5-minute incubation results (one-way analysis of variance). (B) Compartmental distribution of the MDNIC-like metabolite formed following addition of glut-BDNIC to whole blood [hematocrit $(\mathrm{HCT})=33 \% \pm 2 \%$ ] and plasma-free blood (RBCs washed three times with saline and then suspended in saline $[1: 1(\mathrm{v} / \mathrm{v})] ; \mathrm{HCT}=50 \%)$. Where noted, RBCs were separated from plasma/saline by centrifugation. Statistical significance was only evaluated for the 5-minute incubation results (one-way analysis of variance). (C) Molecular weight of DNICs in plasma. Glut-BDNIC, or glut-MDNIC was incubated with plasma for 5 minutes, and then the HMW and LMW fractions were separated by size-exclusion column prior to EPR quantification. DNIC recovery was calculated by dividing the quantity in each fraction by the total amount added. Ten microliters of $2.5 \mathrm{mM}$ glut-BDNIC was added into $990 \mu \mathrm{l}$ of each biomatrix. ${ }^{*} P<0.05$, only comparisons of interests are shown; N/A, not applicable; N.D., not detectable.

rats and sheep, blood pressure was unaffected by administration of comparable doses of glut-BDNIC (Figs. 3 and 4). Such an observation suggests that little SNO was formed in vivo following glut-BDNIC infusion (calculated earlier as $<1 / 8$ of BDNIC infused). Further, EPR results demonstrated nearly complete recovery of both glut-BDNICs and glut-MDNICs as DNICs after their reaction with plasma, excluding the possibility they were converted to SNOs (Fig. 7C). There are several possible explanations for the discrepancy between previous reports suggesting DNICs are converted to SNOs (Boese et al., 1995; Stojanovic et al., 2004; Bosworth et al., 2009; Vanin, 2009; Vanin and Burbaev, 2011) and the lack of evidence in our current work. First, some previous experiments were performed using $\mathrm{NO}^{\circ}$ as the initiator of reactions in the presence of iron and thiols (Stojanovic et al., 2004; Bosworth et al., 2009). In these experiments, DNICs were simply an intermediate product, whereas presynthesized DNICs were used for the current experiments. Therefore, in the previous studies, the SNOs might have been a byproduct of oxidation reactions of the $\mathrm{NO}^{*}$ that was used for synthesizing the DNICs. Others have reported the production of SNOs from cellular DNICs under hypoxic or anoxic conditions, thus minimizing the possibility of $\mathrm{NO}^{\bullet}$ oxidation reactions. However, these previous studies used $\mathrm{HgCl}_{2}$ to selectively detect SNO concentrations, a method recently found to artifactually detect DNICs as SNOs (Keszler et al., 2017). Although recent closer examinations (Hickok et al., 2011, 2012) of this issue agree with our results that production of SNOs from DNICs is not a predominant mode of action of DNICs, if it occurs at all, we would submit that a validated method that selectively detects SNOs in the presence of DNICs is needed to address this question rigorously.

What Are the Hypotensive Effects of BDNIC and the Underlying Mechanisms? The hypotensive effects of glutBDNIC have been described by previous researchers (Lakomkin et al., 2007; Chazov et al., 2012; Borodulin et al., 2013b; Timoshin et al., 2015), leading to the ongoing clinical development of glut-BDNIC for treating hypertension (Chazov et al., 2012). Our results confirm these previous reports by demonstrating a prolonged hypotensive response to high-dose bolus injection (Fig. 6). In the present experiments, the hypotension lasted even longer than comparable doses used in previous reports (Chazov et al., 2012; Borodulin et al., 2013b), perhaps due to sympathetic blockade in our animal model.

Although GSNO and glut-DNICs had similar vasodilatory potencies in isolated arterial rings (Figs. 1 and 4E; Supplemental Fig. S3), their potencies differed markedly in vivo. As calculated in the Results section, GSNO was found to be at least an 8-fold more potent vasodilator than glut-BDNIC in vivo. Notably, glut-BDNIC-induced hypotension lasted for 
more than 4 hours (Fig. 6). Such persistence could be at least partly attributed to the long elimination half-life of the MDNIC-like metabolite derived from glut-BDNIC (Fig. 6D) and is likely influenced by its interaction with plasma albumin as discussed next.

How Might Plasma Alter the Vasoactivity of GlutBDNIC? It has long been proposed that proteins stabilize DNICs (Vanin, 2009; Suryo Rahmanto et al., 2012), possibly attenuating their physiologic activity. Consistent with such stabilization, we found that plasma attenuated glut-BDNICmediated vasodilation both in vitro and in vivo. Other earlier investigators found that MDNIC could exchange its LMW thiolate ligands for either the cysteine-thiol or histidine residues in albumin to form HMW MDNICs (Boese et al., 1995; Timoshin et al., 2007). The present glut-BDNIC experiments support only the involvement of the cysteine-thiol residues, however (Fig. 5D), as the vasoactivity of glut-BDNIC in the presence of albumin was only attenuated by covalent modification of cysteine-thiol residues and not by modification of histidine residues. This finding suggests that glut-BDNIC indeed binds to albumin, possibly via its cysteine-thiol.

Notably, glut-BDNIC and glut-MDNIC added into plasma were both converted into HMW metabolites (Fig. 7C). The EPR spectrum of the HMW metabolite of glut-MDNIC is indistinguishable from that of glut-MDNIC itself. In contrast, although the glut-BDNIC changes from an EPR-silent signal to one that is similar to that of MDNICs, distinct differences in the spectra were noted (Supplemental Fig. 7). These differences may indicate that the HMW metabolites of glut-BDNIC are not physically equivalent to the HMW metabolites of glutMDNIC. Consistent with this view, the significant attenuating effects of plasma and albumin on glut-BDNIC-mediated vasodilation were not observed for glut-MDNIC-induced relaxation (Supplemental Fig. 6). These results suggest that the HMW metabolite of glut-BDNIC is also functionally different from the HMW metabolite of glut-MDNIC. Thus, although glut-BDNIC and glut-MDNIC display similar vasodilatory and HNO-releasing characteristics in vitro, key differences exist between the physical characteristics and vasodilatory effects of their HMW products in plasma, suggesting that further characterization of the structure of the HMW glutBDNIC metabolite is needed before concluding that it is simply an HMW MDNIC.

The reason for the decrease in vasodilatory potency of glutBDNIC upon reacting with albumin is not well understood. One possibility is that the $\mathrm{NO}$ moieties in the $\mathrm{Fe}(\mathrm{NO})_{2}$ core are stabilized when the DNIC is bound to albumin. The decreased vasoactivity would then be a result of a decreased rate of release of HNO from the HMW DNIC. Alternatively, the albumin-bound DNIC may not release HNO, but is instead in equilibrium with a minor fraction of LMW DNIC (Fig. 7C), which is less stable (Timoshin et al., 2015) and thus more likely to release HNO. In either case, the stabilization would prolong the hypotensive effects of DNICs and account for the relatively long biologic half-life of 44 minutes.

Because neither glut-BDNIC nor its HMW metabolites are membrane-permeable (Figs. 6D and 7B), vasodilation is not likely to involve direct interaction between DNICs and sGC. Instead, it may result from the release of HNO from the DNIC outside the cell, which can then diffuse into the smooth muscle cells and activate the sGC either directly (Miller et al., 2009) or after conversion to $\mathrm{NO}^{\bullet}$ (Zeller et al., 2009). In any event, the current experiments do not exclude the possible involvement of an undiscovered vascular smooth muscle membrane transporter or receptor-mediated dilatory signaling pathway that could be stimulated by DNICs. A further possibility is that the hypotensive effects of glut-DNIC result from the calcitonin gene-related peptide which can be induced by HNO via sGC activation (Favaloro and Kemp-Harper, 2007).

Some limitations of the present study should be acknowledged. First, although multiple lines of evidence from both functional and chemical tests support glut-BDNIC as an HNO donor, further investigation with scavengers or probes that can react selectively with $\mathrm{HNO}$ over $\mathrm{NO}^{\bullet}$ is necessary. Although not currently commercially available, such compounds are under active development and validation (Tan et al., 2015; Zhou et al., 2017). Second, depending upon the conditions, the redox state of NO moieties in glut-BDNIC might vary. Thus, we cannot exclude the possibility that DNICs might exhibit distinct biochemical properties under a different environment.

\section{Conclusion}

In sum, the results of this study indicate that glut-BDNIC is an HNO donor and a poor, if at all, $\mathrm{NO}^{\bullet}$ or $\mathrm{NO}^{+}$(SNO) generator. Plasma proteins (such as albumin), via reactions involving its cysteine-thiol residues, modulate glut-BDNICmediated vasodilation in such a way that attenuates its hypotensive potency but prolongs its duration of action.

\section{Acknowledgments}

The authors thank Dr. Jack Lancaster (University of Pittsburgh) for helpful discussion, and Shannon L. Bragg for excellent technical assistance.

\section{Authorship Contributions}

Participated in research design: Liu, Zhang, Schroeder, Wilson, Power, Li, Tipple, Borchardt, Blood.

Conduct Experiments: Liu, Zhang, Terry, Schroeder, Li, Borchardt. Performed data analysis: Liu, Zhang, Terry, Schroeder, Wilson, Power, Li, Borchardt, Blood.

Wrote or contributed to the writing of the manuscript: Liu, Schroeder, Power, Li, Blood.

\section{References}

Blum-Johnston C, Thorpe RB, Wee C, Romero M, Brunelle A, Blood Q, Wilson R, Blood AB, Francis M, Taylor MS, et al. (2016) Developmental acceleration of bradykinin-dependent relaxation by prenatal chronic hypoxia impedes normal development after birth. Am J Physiol Lung Cell Mol Physiol 310:L271-L286.

Boese M, Mordvintcev PI, Vanin AF, Busse R, and Mülsch A (1995) S-nitrosation of serum albumin by dinitrosyl-iron complex. J Biol Chem 270:29244-29249.

Borodulin RR, Dereven'kov IA, Burbaev DSh, Makarov SV, Mikoyan VD, Serezhenkov VA, Kubrina LN, Ivanovic-Burmazovic I, and Vanin AF (2014) Redox activities of mono- and binuclear forms of low-molecular and protein-bound dinitrosyl iron complexes with thiol-containing ligands. Nitric Oxide 40:100-109.

Borodulin RR, Kubrina LN, Mikoyan VD, Poltorakov AP, Shvydkiy VO, Burbaev DSh, Serezhenkov VA, Yakhontova ER, and Vanin AF (2013a) Dinitrosyl iron complexes with glutathione as $\mathrm{NO}$ and $\mathrm{NO}^{+}$donors. Nitric Oxide 29:4-16.

Borodulin RR, Kubrina LN, Shvydkiy VO, Lakomkin VL, and Vanin AF (2013b) A simple protocol for the synthesis of dinitrosyl iron complexes with glutathione: EPR, optical, chromatographic and biological characterization of reaction products. Nitric Oxide 35:110-115.

Bosworth CA, Toledo JC, Jr, Zmijewski JW, Li Q, and Lancaster JR, Jr (2009) Dinitrosyliron complexes and the mechanism(s) of cellular protein nitrosothiol formation from nitric oxide. Proc Natl Acad Sci USA 106:4671-4676.

Chazov EI, Rodnenkov OV, Zorin AV, Lakomkin VL, Gramovich VV, Vyborov ON, Dragnev AG, Timoshin CA, Buryachkovskaya LI, Abramov AA, et al. (2012) Hypotensive effect of oxacom ${ }^{\circledR}$ containing a dinitrosyl iron complex with glutathione: animal studies and clinical trials on healthy volunteers. Nitric Oxide 26:148-156.

Cosby K, Partovi KS, Crawford JH, Patel RP, Reiter CD, Martyr S, Yang BK, Waclawiw MA, Zalos G, Xu X, et al. (2003) Nitrite reduction to nitric oxide by deoxyhemoglobin vasodilates the human circulation. Nat Med 9:1498-1505. 
Favaloro JL and Kemp-Harper BK (2007) The nitroxyl anion (HNO) is a potent dilator of rat coronary vasculature. Cardiovasc Res 73:587-596.

Flores-Santana W, Salmon DJ, Donzelli S, Switzer CH, Basudhar D, Ridnour L, Cheng R, Glynn SA, Paolocci N, Fukuto JM, et al. (2011) The specificity of nitroxyl chemistry is unique among nitrogen oxides in biological systems. Antioxid Redox Signal 14:1659-1674.

Goldstein S, Russo A, and Samuni A (2003) Reactions of PTIO and carboxy-PTIO with *NO, *NO2, and O2-*. J Biol Chem 278:50949-50955.

Hickok JR, Sahni S, Shen H, Arvind A, Antoniou C, Fung LWM, and Thomas DD (2011) Dinitrosyliron complexes are the most abundant nitric oxide-derived cellular adduct: biological parameters of assembly and disappearance. Free Radic Biol Med 51:1558-1566.

Hickok JR, Vasudevan D, Thatcher GR, and Thomas DD (2012) Is S-nitrosocysteine a true surrogate for nitric oxide? Antioxid Redox Signal 17:962-968.

Keszler A, Diers AR, Ding Z, and Hogg N (2017) Thiolate-based dinitrosyl iron complexes: decomposition and detection and differentiation from S-nitrosothiols. Nitric Oxide 65:1-9.

Lakomkin VL, Vanin AF, Timoshin AA, Kapelko VI, and Chazov EI (2007) Longlasting hypotensive action of stable preparations of dinitrosyl-iron complexes with thiol-containing ligands in conscious normotensive and hypertensive rats. Nitric Oxide 16:413-418.

Lee HB and Blaufox MD (1985) Blood volume in the rat. J Nucl Med 26:72-76.

Liu T, Schroeder HJ, Wilson SM, Terry MH, Romero M, Longo LD, Power GG, and Blood $\mathrm{AB}$ (2016a) Local and systemic vasodilatory effects of low molecular weight S-nitrosothiols. Free Radic Biol Med 91:215-223.

Liu T, Schroeder HJ, Zhang M, Wilson SM, Terry MH, Longo LD, Power GG, and Blood $\mathrm{AB}(2016 \mathrm{~b})$ S-nitrosothiols dilate the mesenteric artery more potently than the femoral artery by a cGMP and L-type calcium channel-dependent mechanism. Nitric Oxide 58:20-27.

Miller TW, Cherney MM, Lee AJ, Francoleon NE, Farmer PJ, King SB, Hobbs AJ, Miranda KM, Burstyn JN, and Fukuto JM (2009) The effects of nitroxyl (HNO) on soluble guanylate cyclase activity: interactions at ferrous heme and cysteine thiols. $J$ Biol Chem 284:21788-21796.

Moro MA, Russel RJ, Cellek S, Lizasoain I, Su Y, Darley-Usmar VM, Radomski MW, and Moncada S (1996) cGMP mediates the vascular and platelet actions of nitric oxide: confirmation using an inhibitor of the soluble guanylyl cyclase. Proc Nat Acad Sci USA 93:1480-1485.

Mülsch A, Mordvintcev P, Vanin AF, and Busse R (1991) The potent vasodilating and guanylyl cyclase activating dinitrosyl-iron(II) complex is stored in a protein-bound form in vascular tissue and is released by thiols. FEBS Lett 294:252-256.

Pawloski JR, Hess DT, and Stamler JS (2001) Export by red blood cells of nitric oxide bioactivity. Nature 409:622-626.

Samuni U, Samuni Y, and Goldstein S (2010) On the distinction between nitroxyl and nitric oxide using nitronyl nitroxides. J Am Chem Soc 132:8428-8432.

Severina IS, Bussygina OG, Pyatakova NV, Malenkova IV, and Vanin AF (2003) Activation of soluble guanylate cyclase by NO donors-S-nitrosothiols, and dinitrosyl-iron complexes with thiol-containing ligands. Nitric Oxide 8: $155-163$

Shoman ME and Aly OM (2016) Nitroxyl (HNO): a reduced form of nitric oxide with distinct chemical, pharmacological, and therapeutic properties. Oxid Med Cell Longev 2016:4867124.

Stamler JS, Singel DJ, and Loscalzo J (1992) Biochemistry of nitric oxide and its redox-activated forms. Science 258:1898-1902.
Stojanović S, Stanić D, Nikolić M, Spasić M, and Niketić V (2004) Iron catalyzed conversion of NO into nitrosonium (NO+) and nitroxyl (HNO/NO-) species. Nitric Oxide 11:256-262.

Suryo Rahmanto Y, Kalinowski DS, Lane DJR, Lok HC, Richardson V, and Richardson DR (2012) Nitrogen monoxide (NO) storage and transport by dinitrosyl-dithiol-iron complexes: long-lived NO that is trafficked by interacting proteins. J Biol Chem 287:6960-6968.

Switzer CH, Flores-Santana W, Mancardi D, Donzelli S, Basudhar D, Ridnour LA, Miranda KM, Fukuto JM, Paolocci N, and Wink DA (2009) The emergence of nitroxyl (HNO) as a pharmacological agent. Biochim Biophys Acta 1787:835-840.

Tan Y, Liu RC, Zhang HT, Peltier R, Lam YW, Zhu Q, Hu Y, and Sun HY (2015) Design and synthesis of near-infrared fluorescent probes for imaging of biological nitroxyl. Sci Rep 5:16979.

Timoshin AA, Lakomkin VL, Abramov AA, Ruuge EK, Kapel'ko VI, Chazov EI, and Vanin AF (2015) The hypotensive effect of the nitric monoxide donor Oxacom at different routs of its administration to experimental animals. Eur $J$ Pharmacol 765:525-532.

Timoshin AA, Vanin AF, Orlova TR, Sanina NA, Ruuge EK, Aldoshin SM, and Chazov EI (2007) Protein-bound dinitrosyl-iron complexes appearing in blood of rabbit added with a low-molecular dinitrosyl-iron complex: EPR studies. Nitric Oxide 16:286-293.

Tsai ML, Tsou CC, and Liaw WF (2015) Dinitrosyl iron complexes (DNICs): from biomimetic synthesis and spectroscopic characterization toward unveiling the biological and catalytic roles of DNICs. Acc Chem Res 48:1184-1193.

Tseng YT, Chen CH, Lin JY, Li BH, Lu YH, Lin CH, Chen HT, Weng TC, Sokaras D, Chen HY, et al. (2015) To transfer or not to transfer? Development of a dinitrosyl iron complex as a nitroxyl donor for the nitroxylation of an $\mathrm{Fe}$ (III) -porphyrin center. Chemistry 21:17570-17573.

Vanin AF (2009) Dinitrosyl iron complexes with thiolate ligands: physico-chemistry, biochemistry and physiology. Nitric Oxide 21:1-13.

Vanin AF (2016) Dinitrosyl iron complexes with thiol-containing ligands as a "working form" of endogenous nitric oxide. Nitric Oxide 54:15-29.

Vanin AF and Burbaev DSh (2011) Electronic and spatial structures of water-soluble dinitrosyl iron complexes with thiol-containing ligands underlying their ability to act as nitric oxide and nitrosonium ion donors. J Biophys 2011:878236.

Vanin AF, Mokh VP, Serezhenkov VA, and Chazov EI (2007) Vasorelaxing activity of stable powder preparations of dinitrosyl iron complexes with cysteine or glutathione ligands. Nitric Oxide 16:322-330.

Vanin AF, Stukan RA, and Manukhina EB (1996) Physical properties of dinitrosyl iron complexes with thiol-containing ligands in relation with their vasodilator activity. Biochim Biophys Acta 1295:5-12.

Zeller A, Wenzl MV, Beretta M, Stessel H, Russwurm M, Koesling D, Schmidt K, and Mayer B (2009) Mechanisms underlying activation of soluble guanylate cyclase by the nitroxyl donor Angeli's salt. Mol Pharmacol 76:1115-1122.

Zhou Y, Zhang X, Yang S, Li Y, Qing Z, Zheng J, Li J, and Yang R (2017) Ratiometric visualization of $\mathrm{NO} / \mathrm{H}_{2} \mathrm{~S}$ cross-talk in living cells and tissues using a nitroxylresponsive two-photon fluorescence probe. Anal Chem 89:4587-4594.

Address correspondence to: Arlin Blood, Department of Pediatrics, and Center for Perinatal Biology, Loma Linda University, 11175 Campus Street, 11121 Coleman, Loma Linda, CA 92354. E-mail: ablood@llu.edu 